\section{Delayed Huntington's Disease Diagnosis in Two Alcoholic Patients With a Family History of "Parkinson's Disease"}

To the Editor: Huntington's disease is an autosomal dominant neurodegenerative disease characterized by a triad of symptoms and signs including movement disorder, cognitive impairment, and behavioral syndromes. ${ }^{1}$ As the earliest pathological changes in Huntington's disease are in the associative portion of the striatum, the domains first affected by the disease may be cognitive and psychiatric rather than motor. ${ }^{2,3}$

\section{Case Report}

A 51-year-old woman with a history of alcohol abuse, though abstinent for 3 years, presented with cognitive deterioration. She could no longer teach properly because she forgot what to say, was unable to repeat information, could no longer handle money, and didn't know how to cook or take care of the house. On one occasion she tried to spice the salad with liquid soap and put the mobile phone in the refrigerator. She also complained of irritability, aggressive outbursts, sadness, anxiety, restlessness, and hyperphagia with a preference for sweet foods. These symptoms evolved over the last 10 years. In the previous 3 years, after being hospitalized for alcohol detoxification, she started noticing unsteady gait with frequent falls and involuntary movements, especially in her lower limbs, which would frequently kick even when she was lying down.
Her family history included what was called "Parkinson's disease" in her mother, grandmother and two maternal uncles. The patient also had a 52-year-old sister who was receiving psychiatric treatment for alcohol abuse and depressive syndrome and who had recent presented behavioral changes including agitation, anxiety, overactivity, and frequent falls.

Neurological examination of the patient showed severe akathisia with noticeable lower-limb predominant choreiform movements and discrete motor perseveration. Her Mini-Mental State Examination score was 23.

An analytic study, including CBC, vitamin $B_{12}$ and folic acid levels, thyroid, and hepatic functions revealed no abnormalities in our patient. A brain MRI showed generalized brain atrophy especially in the frontal lobe. Neuropsychological study revealed dementia in an early stage with frontotemporal characteristics. Genetic testing for Huntington's disease was positive, as it was also positive for our patient's oldest sister. The patient's daughter decided to do the presymptomatic test for Huntington's disease and had also the abnormal expansion in the gene.

\section{Discussion}

Glutamate excitotoxicity has been implicated in the pathophysiology of movement disorder and other manifestations of Huntington's disease. Alcohol has been shown to inhibit NMDA receptors in rats and mice as well as to retard progression of Huntington's disease disorder in humans. ${ }^{4}$ Chronic NMDA inhibition has also been reported to lead to NMDA upregulation, increasing the risk of glutamate excitotoxicity. ${ }^{4}$ Thus, alcohol intake may initially inhibit NMDA receptors and mask Huntington's disease manifestations, but chronic alcohol use may lead to receptor upregulation, resulting in NMDA excitotoxicity and manifestation of Huntington's disease. ${ }^{4}$

Huntington's disease patients can present with rigidity without signs of chorea. Such individuals can be misdiagnosed with Parkinson's disease, catatonia, or schizophrenia. ${ }^{5}$ This might have been the case with this patient's mother and grandmother, both of whom died with cognitive deterioration and movement abnormalities.

This case highlights the importance of a careful family history, as the presence of disease in multiple members of the same family can be a fundamental clue for a genetic cause and help to make the correct diagnosis.

Joana Mesquita, M.D.

Luísa Silva, M.D.

Psychiatry and Mental Health

Department, Hospital de São

Marcos, Braga, Portugal

Álvaro Machado, M.D.

Neurology Department, Hospital

de São Marcos, Braga, Portugal

\section{References}

1. Anderson KE, Marder KS: An overview of psychiatric symptoms in Huntington's disease. Curr Psychiatr Rep 2001; 3:379-388

2. Paulsen JS, Ready RE, Hamilton JM, et al: Neuropsychiatric aspects of Huntington's disease. J Neurol Neurosurg Psychiatry 2001; 71:310-314

3. Duijn EV, Kingma EM, Mast RC: Psychopathology in verified Huntington's disease gene carriers. J Neuropsychiatry Clin Neurosci 2007; 19:441-447

4. Matoo SK, Khurana H: Huntington's disease and alcohol abuse. Neurol India 1999; 47:68-70

5. Walker FO: Huntington's disease. Lancet 2007; 369:218-228 